\title{
Chapter 18 \\ Long-Time Behavior of State Functions for Badyko Models
}

\author{
Nataliia V. Gorban, Mark O. Gluzman, Pavlo O. Kasyanov \\ and Alla M. Tkachuk
}

\begin{abstract}
In this note we examine the long-time behavior of state functions for a climate energy balance model (Budyko Model) in the strongest topologies of the phase and the extended phase spaces. Strongest convergence results for all weak solutions are obtained. New structure and regularity properties for global and trajectory attractors are justified.
\end{abstract}

\subsection{Introduction and Setting of the Problem}

Let $(\mathscr{M}, \mathbf{g})$ be a $C^{\infty}$ compact connected oriented two-dimensional Riemannian manifold without boundary (e.g., $\mathscr{M}=S^{2}$ the unit sphere of $\mathbb{R}^{3}$ ). Consider the problem:

$$
\frac{\partial u}{\partial t}-\Delta u+R_{e}(x, u) \in Q S(x) \beta(u), \quad(x, t) \in \mathbb{R}_{+} \times \mathscr{M},
$$

where $\Delta u=\operatorname{div} \mathscr{M}\left(\nabla_{\mathscr{M}} u\right) ; \nabla_{\mathscr{M}}$ is understood in the sense of the Riemannian metric g. Note that (18.1) is the so-called climate energy balance model. It was proposed in Budyko [4] and Sellers [38] and examined also in Díaz et al. [10-13]. The unknown

\footnotetext{
N.V. Gorban · P.O. Kasyanov

Institute for Applied System Analysis, National Technical University of Ukraine "Kyiv Polytechnic Institute", Peremogy ave., 37, build, 35, Kyiv 03056, Ukraine e-mail: nata_gorban@i.ua

P.O. Kasyanov

e-mail: kasyanov@i.ua

M.O. Gluzman ( $₫)$

Department of Applied Physics and Applied Mathematics,

Columbia University, New York, NY 10027, USA

e-mail: mark.gluzman@columbia.edu

A.M. Tkachuk

Faculty of Automation and Computer Systems,

National University of Food Technologies, Volodymyrska st., 68, Kyiv 01601, Ukraine

e-mail: tkachukam@ukr.net 
$u(x, t)$ represents the average temperature of the Earth's surface. In Budyko [4] the energy balance is expressed as

$$
\text { heat variation }=R_{a}-R_{e}+D \text {. }
$$

Here $R_{a}=Q S(x) \beta(u)$. It represents the solar energy absorbed by the Earth, $Q>0$ is a solar constant, $S(x)$ is an insolation function (the distribution of solar radiation falling on upper atmosphere), $\beta$ represents the ratio between absorbed and incident solar energy at the point $x$ of the Earth's surface (so-called the co-albedo function). The term $R_{e}$ represents the energy emitted by the Earth into space, and as usual, it is assumed to be an increasing function on $\mathrm{u}$. The term $D$ is the heat diffusion, and we assume (for simplicity) that it is constant.

As usual, the term $R_{e}$ may be chosen according to the Newton cooling law as linear function on $u, R_{e}=B u+C$ (here $B$ and $C$ are some positive constants) [4], or according to the Stefan-Boltzmann law, $R_{e}=\sigma u^{4}$ [38]. In this note we consider $R_{e}=B u$ as in Budyko [4].

Let $S: \mathscr{M} \rightarrow \mathbb{R}$ be a function such that $S \in L^{\infty}(\mathscr{M})$, and there exist $S_{0}, S_{1}>0$ such that

$$
0<S_{0} \leq S(x) \leq S_{1}
$$

Suppose also that $\beta$ is a bounded maximal monotone graph of $\mathbb{R}^{2}$; that is, there exist $m, M \in \mathbb{R}$, such that for all $s \in \mathbb{R}$ and $z \in \beta(s)$

$$
m \leq z \leq M
$$

Through the note we consider real Hilbert spaces

$$
H:=L^{2}(\mathscr{M}), \quad V:=\left\{u \in L^{2}(\mathscr{M}): \nabla_{\mathscr{M}} u \in L^{2}(T \mathscr{M})\right\}
$$

with respective standard norms $\|\cdot\|_{H},\|\cdot\|_{V}$, and inner products $(\cdot, \cdot)_{H},(\cdot, \cdot)_{V}$, where $T \mathscr{M}$ represents the tangent bundle and the functional spaces $L^{2}(\mathscr{M})$ and $L^{2}(T \mathscr{M})$ are defined in a standard way; see, for example, Aubin [2]. Let $V^{*}$ be the dual space of the function space $V$. We remark that

$$
V \subset H \subset V^{*}
$$

and all embeddings are compact and dense; see, for example, Aubin [2, p. 55, Theorem 2.34].

Let $-\infty<\tau<T<+\infty$. A function $u(\cdot) \in L^{2}(\tau, T ; V)$ is called a weak solution of Problem (18.1) on [ $\tau, T]$, if there exists a measurable function $d: \mathscr{M} \times(\tau, T) \rightarrow$ $\mathbb{R}$ such that

$$
d(x, t) \in Q S(x) \beta(u(x, t)) \text { for a.e. }(x, t) \in \mathscr{M} \times(\tau, T),
$$


and

$$
\int_{\tau}^{T}\left[\left\langle-u, \frac{\partial \xi}{\partial t}\right\rangle-\langle u, \triangle \xi\rangle+\left\langle R_{e}(\cdot, t, u), \xi\right\rangle-\langle d, \xi\rangle\right] d t=0
$$

for all $\xi \in C_{0}^{\infty}(\mathscr{M} \times(\tau, T))$, where $\langle\cdot, \cdot\rangle$ denotes the pairing in the space $V$.

In this manuscript, we examine the long-term dynamics as $t \rightarrow+\infty$ of all weak solution for Problem (18.1) in the strongest sense under the assumptions listed above.

We note that the existence of a Lyapunov function for a class of semi-linear parabolic differential reaction-diffusion equations with discontinuous nonlinearities, regularity properties for global and trajectory attractors, and its applications were considered in [16-18]. In [5, 32, 46, 48, 49] authors provided sufficient conditions for the existence of a Lyapunov function for autonomous evolution inclusions of hyperbolic type. The theory of the global and trajectory attractors for parabolic systems in the natural phase and extended phase spaces was considered in $[1,3,6-9$, 14, 19-28, 30, 31, 33, 39-45]. Topological properties of strong and weak solutions were provided in [15, 34-37]. Strong regularity properties of global and trajectory attractors were proved in [10, 26-29].

\subsection{Auxiliaries}

According to [16], for each $u_{0} \in H$ and $T>0$, there exists at least one weak solution of Problem (18.1) on [0,T]. Moreover, each weak solution $u(\cdot)$ of Problem (18.1) on $[0, T]$ is regular, that is, $u(\cdot) \in C([\varepsilon, T] ; V) \cap L^{2}(\varepsilon, T ; D(A))$ and $u_{t}(\cdot) \in L^{2}(\varepsilon, T ; H)$, for each $\varepsilon \in(0, T)$; see Gluzman et al. [16, Theorem 14.1], where $D(A):=\{u \in V: A u \in H\}$ and $\langle A u, v\rangle_{V}=(u, v)_{V}$ for each $u, v \in V$. Furthermore, each weak solution of Problem (18.1) on $[0, T]$ can be extended to a global one defined on $[0,+\infty)$; see Gluzman et al. [16, p. 235].

Denote by $\mathscr{D}\left(u_{0}\right)$ the set of all weak solutions of Problem (18.1) globally defined on $[0,+\infty)$ with initial data $u(0)=u_{0}, u_{0} \in H$. Then, $\mathscr{D}\left(u_{0}\right) \subset L_{l o c}^{2}(0,+\infty ; V) \cap$ $C([0,+\infty), H)$ for each $u_{0} \in H$. Moreover, $\mathscr{D}\left(u_{0}\right) \subset L^{\infty}(0,+\infty ; H)$ for each $u_{0} \in$ $H$.

Consider the family of all weak solutions of Problem (18.1) defined on the semiinfinite time interval $[0,+\infty)$ :

$$
\mathscr{K}=\cup_{u_{0} \in H} \mathscr{D}\left(u_{0}\right)
$$

The set $\mathscr{K}_{+}$is a translation invariant one, that is, $u(\cdot+h) \in \mathscr{K}_{+}$for each $u(\cdot) \in \mathscr{K}_{+}$ and $h \geq 0$.

Let us consider Problem (18.1) on the entire time axis. A function $u \in L^{\infty}(\mathbb{R} ; H)$ is called a complete trajectory of Problem (18.1), if $\Pi_{+} u(\cdot+h) \in \mathscr{K}_{+}$for each $h \geq 0$, where $\Pi_{+}$is the restriction operator to the interval $[0,+\infty)$. Denote by $\mathscr{K}$ the family of all complete trajectories of Problem (18.1) A complete trajectory 
$u(\cdot) \in \mathscr{K}$ is stationary if there is $z \in D(A)$ such that $u(t)=z$ for all $t \in \mathbb{R}$. Such $z$ is called a rest point. We denote the set of all rest points by $Z$.

Definition 18.1 The function $E: V \rightarrow \mathbb{R}$ is called a Lyapunov type one for $\mathscr{K}_{+}$, if the following conditions hold:

(a) $E$ is continuous on $V$;

(b) $E(u(t)) \leq E(u(s))$ whenever $u \in \mathscr{K}_{+}$and $t \geq s>0$;

(c) If $E(u(\cdot)) \equiv$ const, for some $u \in \mathscr{K}$, then $u$ is stationary complete trajectory.

Let $\Upsilon(s)$ be a real function such that $\partial \Upsilon(s)=\beta(s)$ for each $s \in \mathbb{R}$ and $\mathbf{1}: \mathscr{M} \rightarrow$ $\mathbb{R}, \mathbf{1} \equiv 1$. According to Gluzman et al. [16, Theorem 14.2], the following function

$$
E(u)=\frac{1}{2}\|u\|_{V}^{2}+\frac{B}{2}\|u\|_{H}^{2}-Q\langle S(\cdot) \Upsilon(u), \mathbf{1}\rangle \quad u \in V,
$$

is a Lyapunov-type function for $\mathscr{K}_{+}$. Moreover, the following energy equality holds:

$$
E(u(T))-E(u(\tau))=-\int_{\tau}^{T}\left\|\frac{\partial u}{\partial s}(\cdot, s)\right\|_{H}^{2} d s,
$$

for each $u \in \mathscr{K}_{+}$and $0<\tau<T<\infty$. The following lemma provides the main convergence result for all weak solutions of Problem (18.1) in the strongest topologies.

Lemma 18.1 (Gluzman et al. [16, Theorem 14.3]) Let $0<\tau<T, u_{\tau} \in H$, and $\left\{u_{n}(\cdot)\right\}_{n \geq 1}$ be a sequence of weak solutions for Problem (18.1) on $[\tau, T]$. Furthermore, let $u_{n}(\tau) \rightarrow u_{\tau}$ weakly in $H$ as $n \rightarrow \infty$. Then, there exists a weak solution $u(\cdot)$ for Problem (18.1) on $[\tau, T]$ such that $u(\tau)=u_{\tau}$, and there exists an increasing sequence of positive integers $\left\{n_{k}\right\}_{k \geq 1}$ such that for each $\varepsilon \in(0, T-\tau)$

$$
\sup _{t \in[\tau+\varepsilon, T]}\left\|u_{n_{k}}(t)-u(t)\right\|_{V}+\int_{\tau+\varepsilon}^{T}\left\|\frac{\partial u_{n_{k}}}{\partial s}(\cdot, s)-\frac{\partial u}{\partial s}(\cdot, s)\right\|_{H}^{2} d s \rightarrow 0,
$$

as $k \rightarrow+\infty$.

Definition 18.2 The multivalued map $G: \mathbb{R}_{+} \times H \rightarrow 2^{H} \backslash \emptyset$ is called a strict multivalued semiflow if:

(a) $G(0, \cdot)=$ Id (the identity map);

(b) $G(t+s, x)=G(t, G(s, x)) \forall x \in H, t, s \in \mathbb{R}_{+}$.

Let us define the multivalued map $G: \mathbb{R}_{+} \times H \rightarrow 2^{H} \backslash\{\emptyset\}$ as follows:

$$
G\left(t, u_{0}\right)=\left\{u(t) \mid u(\cdot) \in \mathscr{K}_{+}, u(0)=u_{0}\right\} .
$$

Lemma 18.2 (Zgurovsky et al. [47, Chap. 2]) The multivalued map $G: \mathbb{R}_{+} \times H \rightarrow$ $2^{H} \backslash\{\emptyset\}$, defined in (18.7), is a strict multivalued semiflow. 


\subsection{Main Results}

In this section we state that there exist trajectory and global attractors for all weak solutions of Problem (18.1) and provide their structure and regularity properties.

Definition 18.3 A set $\mathscr{A} \subseteq H$ is called an invariant global attractor for multivalued semiflow $G$ if the following conditions hold:

(1) $\mathscr{A}$ is an invariant set, that is $\mathscr{A}=G(t, \mathscr{A})$ for each $t \geq 0$;

(2) $\mathscr{A}$ is an attracting set, that is, for each nonempty bounded subset $B \subset H$,

$$
\operatorname{dist}_{H}(G(t, B), \mathscr{A}) \rightarrow 0, \quad t \rightarrow+\infty,
$$

where $\operatorname{dist}_{H}(C, D)=\sup _{c \in C} \inf _{d \in D}\|c-d\|_{H}$ denote the Hausdorff semidistance between nonempty subsets $C$ and $D$ of space $H$.

(3) For any closed attracting set $Y \subseteq H$, we have $\mathscr{A} \subseteq Y$.

Theorem 18.1 The strict multivalued semiflow $G: \mathbb{R}_{+} \times H \rightarrow 2^{H} \backslash \emptyset$, defined in (18.7), has a compact invariant global attractor $\mathscr{A}$ in the phase space $H$.

Let $\{T(h)\}_{h \geq 0}$ be the translation semigroup acting on $\mathscr{K}_{+}$, that is, $T(h) u(\cdot)=$ $u(\cdot+h), h \geq 0, u(\cdot) \in \mathscr{K}_{+}$. On $\mathscr{K}_{+}$, we consider the topology induced from the Fréchet space $C_{\text {loc }}\left(\mathbb{R}_{+} ; H\right)$. Note that $f_{n}(\cdot) \rightarrow f(\cdot)$ in $C_{\text {loc }}\left(\mathbb{R}_{+} ; H\right)$ as $n \rightarrow \infty$ if and only if $\forall M>0 \Pi_{0, M} f_{n}(\cdot) \rightarrow \Pi_{0, M} f(\cdot)$ in $C([0, M] ; H)$ as $n \rightarrow \infty$.

Definition 18.4 A set $\mathscr{U} \subset \mathscr{K}_{+}$is called a trajectory attractor for translation semigroup $\{T(h)\}_{h \geq 0}$ on $\mathscr{K}_{+}$in the induced topology of $C_{\text {loc }}\left(\mathbb{R}_{+} ; H\right)$, if $\mathscr{U} \subset \mathscr{K}_{+}$is a global attractor for the translation semigroup $\{T(h)\}_{h \geq 0}$ acting on $\mathscr{K}_{+}$; see Kasyanov et al. [29, Sect. 3].

Theorem 18.2 There exists a trajectory attractor $\mathscr{U}$ for $\{T(h)\}_{h \geq 0}$ on $\mathscr{K}_{+}$in the induced topology of $C_{\text {loc }}\left(\mathbb{R}_{+} ; H\right)$. Moreover, the following equalities hold:

$$
\mathscr{U}=\Pi_{+} \mathscr{K}=\left\{u(\cdot) \in \mathscr{K}_{+} \mid u(t) \in \mathscr{A} \forall t \in \mathbb{R}_{+}\right\}=\left\{u(\cdot) \in \mathscr{K}_{+} \mid u(0) \in \mathscr{A}\right\}
$$

The following theorem provides structure and regularity properties for global and trajectory attractors for all weak solutions of Problem (18.1).

Theorem 18.3 The following statements hold:

(i) $\mathscr{A}$ is a compact subset of $V$;

(ii) $\mathscr{U}$ is a bounded subset of $L^{\infty}\left(\mathbb{R}_{+} ; V\right)$ and $\Pi_{0, M} \mathscr{U}$ is a compact subset of $W(0, M)$ for each $M>0$, where $W(0, M)=\left\{u(\cdot) \in C([0, M] ; V): u_{t}(\cdot) \in\right.$ $\left.L^{2}(0, M ; H)\right\}$ is a real Banach space;

(iii) $\mathscr{K}$ is a bounded subset of $L^{\infty}(\mathbb{R} ; V)$ and $\Pi_{0, M} \mathscr{U}$ a compact subset of $W(0, M)$ for each $M>0$; 
(iv) For each nonempty bounded set $B \subset H$ dist $_{V}(G(t, B), \mathscr{A}) \rightarrow 0, t \rightarrow \infty$;

(v) For any bounded in $L^{\infty}\left(\mathbb{R}_{+} ; H\right)$ set $\mathbf{B} \subset \mathscr{K}_{+}$and any $M \geq 0$ the following relation holds: dist ${ }_{W(0, M)}\left(\Pi_{0, M} T(t) \mathbf{B}, \Pi_{0, M} \mathscr{U}\right) \rightarrow 0, \quad t \rightarrow+\infty$;

(vi) For each $u \in \mathscr{K}$ the limit sets

$$
\begin{aligned}
& \alpha(u)=\left\{z \in V \mid u\left(t_{j}\right) \rightarrow z \text { in } V \text { for some sequence } t_{j} \rightarrow-\infty\right\}, \\
& \omega(u)=\left\{z \in V \mid u\left(t_{j}\right) \rightarrow z \text { in } V \text { for some sequence } t_{j} \rightarrow+\infty\right\}
\end{aligned}
$$

are connected subsets of $Z$ on which $E$ is constant. If $Z$ is totally disconnected (in particular, if $Z$ is countable) the limits in $V$

$$
z_{-}=\lim _{t \rightarrow-\infty} u(t), \quad z_{+}=\lim _{t \rightarrow+\infty} u(t)
$$

exist and $z_{-}, z_{+}$are rest points; furthermore, $u(t)$ tends in $V$ to a rest point as $t \rightarrow+\infty$ for every $u \in \mathscr{K}_{+}$.

\subsection{Proof of Theorems $18.1,18.2$ and 18.3}

Gluzman et al. [16, Theorem 14.4] yield all the statements of Theorems 18.1, 18.2, and 18.3 , because the spaces $V, H$ and operators $A, J_{1}(\cdot):=\frac{B}{2}\|\cdot\|_{H}^{2}, J_{2}(\cdot):=$ $E(\cdot)-\frac{B}{2}\|\cdot\|_{H}^{2}-\frac{1}{2}\|\cdot\|_{V}^{2}$ satisfy the assumptions of [16, Theorem 14.4], that is,

(a) $\left(V ; H ; V^{*}\right)$ is an evolution triple, where $V$ is a real Hilbert space, such that $V \subset H$ with compact imbedding;

(b) $A: V \rightarrow V^{*}$ is a linear symmetric operator such that there exists $c>0$ such that $\langle A v, v\rangle \geq c\|v\|_{V}^{2}$, for each $v \in V$

(c) $J_{i}: H \rightarrow \mathbb{R}$ is a convex, lower semicontinuous function such that the following assumptions hold: (i) (growth condition) There exists $c_{1}>0$ such that $\|y\|_{H} \leq$ $c_{1}\left(1+\|u\|_{H}\right)$, for each $u \in H$ and $y \in \partial J_{i}(u)$ and $i=1,2$; (ii) (sign condition) there exist $c_{2}>0, \lambda \in(0, c)$ such that $\left(y_{1}-y_{2}, u\right)_{H} \geq-\lambda\|u\|_{H}^{2}-c_{2}$, for each $y_{i} \in \partial J_{i}(u), u \in H$, where $\partial J_{i}(u)$ the subdifferential of $J_{i}(\cdot)$ at a point $u ; i=1,2$, $0<\lambda<\lambda_{1}, \lambda_{1}$ is a first eigenvalue of $A$. Note that $u^{*} \in \partial J_{i}(u)$ if and only if $u^{*}(v-u) \leq J_{i}(v)-J_{i}(u) \forall v \in H ; i=1,2$.

Acknowledgments This work was partially supported by the Ukrainian State Fund for Fundamental Researches under grant GP/F66/14921 and by the National Academy of Sciences of Ukraine under grant 2284 . 


\section{References}

1. Arrieta, J.M., Rodrígues-Bernal, A., Valero, J.: Dynamics of a reaction-diffusion equation with discontinuous nonlinearity. Int. J. Bifurc. Chaos 16, 2695-2984 (2006)

2. Aubin, T.: Nonlinear Analysis on Manifolds. Monge-Ampére Equations. Springer, Berlin (1980)

3. Balibrea, F., Caraballo, T., Kloeden, P.E., Valero, J.: Recent developments in dynamical systems: three perspectives. Int. J. Bifurc. Chaos (2010). doi:10.1142/S0218127410027246

4. Budyko, M.I.: The effects of solar radiation variations on the climate of the Earth. Tellus 21, 611-619 (1969)

5. Ball, J.M.: Global attractors for damped semilinear wave equations. DCDS 10, 31-52 (2004)

6. Barbu, V.: Nonlinear Semigroups and Differential Equations in Banach Spaces. Editura Academiei, Bucuresti (1976)

7. Chepyzhov, V.V., Vishik, M.I.: Trajectory and global attractors of three-dimensional NavierStokes systems. Math. Notes 71, 177-193 (2002). doi:10.1023/A:1014190629738

8. Chepyzhov, V.V., Vishik, M.I.: Trajectory attractor for reaction-diffusion system with diffusion coefficient vanishing in time. Discret. Contin. Dyn. Syst. Ser. A 27, 1493-1509 (2013)

9. Chepyzhov, V.V., Conti, M., Pata, V.: A minimal approach to the theory of global attractors. Discret. Contin. Dyn. Syst. 32, 2079-2088 (2012)

10. Díaz, H., Díaz, J.: On a stochastic parabolic PDE arising in climatology. Rev. R. Acad. Cien. Serie A Mat. 96, 123-128 (2002)

11. Díaz, J., Tello, L.: Infinitely many stationary solutions for a simple climate model via a shooting method. Math. Methods Appl. Sci. 25, 327-334 (2002)

12. Díaz, J., Hernández, J., Tello, L.: On the multiplicity of equilibrium solutions to a nonlinear diffusion equation on a manifold arising in climatology. J. Math. Anal. Appl. 216, 593-613 (1997)

13. Díaz, J., Hernández, J., Tello, L.: Some results about multiplicity and bifurcation of stationary solutions of a reaction diffusion climatological model. Rev. R. Acad. Cien. Serie A. Mat. 96(3), 357-366 (2002)

14. Feireisl, E., Norbury, J.: Some existence and nonuniqueness theorems for solutions of parabolic equations with discontinuous nonlinearities. Proc. R. Soc. Edinb. A. 119(1-2), 1-17 (1991)

15. Gajewski, H., Gröger, K., Zacharias, K.: Nichtlineare operatorgleichungen und operatordifferentialgleichungen. Akademie-Verlag, Berlin (1974)

16. Gluzman, M.O., Gorban, N.V., Kasyanov, P.O.: Lyapunov functions for differential inclusions and applications in physics, biology, and climatology. Continuous and distributed systems II. Theory and applications. Series studies in systems. Decis. Control 30, 233-243 (2015). doi:10. 1007/978-3-319-19075-4_14

17. Gluzman, M.O., Gorban, N.V., Kasyanov, P.O.: Lyapunov type functions for classes of autonomous parabolic feedback control problems and applications. Appl. Math. Lett. (2015). https://dx.doi.ord/10.1016/j.aml.2014.08.006

18. Gluzman, M.O., Gorban, N.V., Kasyanov, P.O.: Lyapunov functions for weak solutions of reaction-diffusion equations with discontinuous interaction functions and its applications. Nonautonomous Dyn. Syst. (2015). doi:10.1515/msds-2015-0001

19. Goldstein, G.R., Miranville, A.: A Cahn-Hilliard-Gurtin model with dynamic boundary conditions. Discret. Contin. Dyn. Syst. Ser. S 6, 387-400 (2013)

20. Gorban, N.V., Kasyanov, P.O.: On regularity of all weak solutions and their attractors for reaction-diffusion inclusion in unbounded domai. Solid Mech. Appl. 211, 205-220 (2014)

21. Gorban, N.V., Kapustyan, O.V., Kasyanov, P.O.: Uniform trajectory attractor for nonautonomous reaction-diffusion equations with Caratheodory's nonlinearity. Nonlinear Anal. Theory Methods Appl. 98, 13-26 (2014). doi:10.1016/j.na.2013.12.004

22. Gorban, N.V., Kapustyan, O.V., Kasyanov, P.O., Paliichuk, L.S.: On global attractors for autonomous damped wave equation with discontinuous nonlinearity. Solid Mech. Appl. 211, $221-237(2014)$ 
23. Efendiev, M., Miranville, A., Zelik, S.: Exponential attractors for a nonlinear reaction-diffusion system in $R^{3}$. Comptes Rendus de l'A Academie des Sciences-Series I - Mathematics 330, 713 $718(2000)$

24. Kalita, P., Lukaszewicz, G.: Global attractors for multivalued semiflows with weak continuity properties. Nonlinear Anal. Theory Methods Appl. 101, 124-143 (2014)

25. Kalita, P., Lukaszewicz, G.: Attractors for Navier-Stokes flows with multivalued and nonmonotone subdifferential boundary conditions. Nonlinear Anal. Real World Appl. 19, 75-88 (2014)

26. Kapustyan, O.V., Kasyanov, P.O., Valero, J.: Regular solutions and global attractors for reactiondiffusion systems without uniqueness. Commun. Pure Appl. Anal. 13, 1891-1906 (2014). doi:10.3934/cpaa.2014.13.1891

27. Kapustyan, O.V., Kasyanov, P.O., Valero, J.: Structure and regularity of the global attractor of a reaction-diffusion equation with non-smooth nonlinear term. Commun. Pure Appl. Anal. 34, 4155-4182 (2014). doi:10.3934/dcds.2014.34.4155

28. Kapustyan, O.V., Kasyanov, P.O., Valero, J., Zgurovsky, M.Z.: Structure of uniform global attractor for general non-autonomous reaction-diffusion system. Solid Mech. Appl. 211, 163180 (2014)

29. Kasyanov, P.O., Toscano, L., Zadoianchuk, N.V.: Regularity of weak solutions and their attractors for a parabolic feedback control problem. Set-Valued Var. Anal. 21, 271-282 (2013). doi:10.1007/s11228-013-0233-8

30. Kasyanov, P.O.: Multivalued dynamics of solutions of an autonomous differential-operator inclusion with pseudomonotone nonlinearity. Cybern. Syst. Anal. 47, 800-811 (2011)

31. Kasyanov, P.O.: Multivalued dynamics of solutions of autonomous operator differential equations with pseudomonotone nonlinearity. Math. Notes 92, 205-218 (2012)

32. Kasyanov, P.O., Toscano, L., Zadoianchuk, N.V.: Long-time behaviour of solutions for autonomous evolution hemivariational inequality with multidimensional "reactiondisplacement" law. Abstr. Appl. Anal. 2012, 21 (2012). doi:10.1155/2012/450984

33. Melnik, V.S., Valero, J.: On attractors of multivalued semiflows and differential inclusions. Set Valued Anal. 6, 83-111 (1998). doi:10.1023/A:1008608431399

34. Migórski, S.: On the existence of solutions for parabolic hemivariational inequalities. J. Comput. Appl. Math. 129, 77-87 (2001)

35. Migórski, S., Ochal, A.: Optimal control of parabolic hemivariational inequalities. J. Glob. Optim. 17, 285-300 (2000)

36. Otani, M., Fujita, H.: On existence of strong solutions for $\frac{d u}{d t}(t)+\partial \varphi^{1}(u(t))-\partial \varphi^{2}(u(t)) \ni$ $f(t)$. J. Fac. Sci. The University of Tokyo. Sect. 1 A, Mathematics. 24(3), 575-605 (1977)

37. Panagiotopoulos, P.D.: Inequality Problems in Mechanics and Applications. Convex and Nonconvex Energy Functions. Birkhauser, Basel (1985)

38. Sellers, W.D.: A global climatic model based on the energy balance of the Earth-atmosphere system. J. Appl. Meteorol. 8, 392-400 (1969)

39. Sell, G.R., You, Y.: Dynamics of Evolutionary Equations. Springer, New York (2002)

40. Temam, R.: Infinite-Dimensional Dynamical Systems in Mechanics and Physics. Springer, New York (1988)

41. Terman, D.: A free boundary problem arising from a bistable reaction diffusion equation. SIAM J. Math. Anal. 14, 1107-1129 (1983)

42. Terman, D.: A free boundary arising from a model for nerve conduction. J. Differ. Equ. 58(3), 345-363 (1985)

43. Valero, J.: Attractors of parabolic equations without uniqueness. J. Dyn. Differ. Equ. 13, 711744 (2001). doi:10.1023/A:1016642525800

44. Valero, J., Kapustyan, A.V.: On the connectedness and asymptotic behaviour of solutions of reaction-diffusion systems. J. Math. Anal. Appl. (2006). doi:10.1016/j.jmaa.2005.10.042

45. Vishik, M.I., Zelik, S.V., Chepyzhov, V.V.: Strong trajectory attractor for dissipative reactiondiffusion system. Doclady Math. (2010). doi:10.1134/S1064562410060086

46. Zadoianchuk, N.V., Kasyanov, P.O.: Dynamics of solutions of a class of second-order autonomous evolution inclusions. Cybern. Syst. Anal. 48, 414-428 (2012) 
47. Zgurovsky, M.Z., Kasyanov, P.O., Kapustyan, O.V., Valero, J., Zadoianchuk, N.V.: Evolution Inclusions and Variation Inequalities for Earth Data Processing III. Springer, Berlin (2012). doi:10.1007/978-3-642-28512-7

48. Zgurovsky, M.Z., Kasyanov, P.O.: Multivalued dynamics of solutions for autonomous operator differential equations in strongest topologies. Solid Mech. Appl. 211, 149-162 (2014)

49. Zgurovsky, M.Z., Kasyanov, P.O., Zadoianchuk, N.V.: Long-time behavior of solutions for quasilinear hyperbolic hemivariational inequalities with application to piezoelectricity problem. Appl. Math. Lett. 25, 1569-1574 (2012). doi:10.1016/j.aml.2012.01.016 